\title{
TEMPOROMANDIBULAR JOINT DISORDERS AND MALOCCLUSION A CROSS-SECTIONAL STUDY
}

\author{
Noha Hussein Abbas* and Dina Hussien El-Ghoul*
}

\begin{abstract}
Objective: This study aimed to investigate the probable association between different types of TMDs and different types of malocclusion.

Materials and methods: One hundred participants complaining of TMJ problem were recruited from the clinic of maxillofacial department, faculty of dentistry, Ain shams university. The type of TMD was diagnosed and evaluated by RDC and the orthodontic examination was performed for each patient to reveal the static and functional occlusal conditions.

Results: The results showed high association between static and functional malocclusion group and TMD patients having combined muscle and joint problems.

Conclusion: The type of TMD whether muscle or joint problem is an important factor when correlating TMD to malocclusion. Combined muscle and joint problems are correlated to malocclusion of combined static and functional occlusal problems.
\end{abstract}

\section{INTRODUCTION}

Temporomandibular disorder (TMD) is a collective term that enfolds a number of clinical problems that involves the masticatory muscles, the temporomandibular joint (TMJ) and the associated structures. The prevalence of temporomandibular disorders has been reported to be high and most often ranges between $10 \%$ to $70 \%$ in general population with a greater prevalence in older people. ${ }^{1}$ It is a main cause of pain of non dental origin in the orofacial region including the head, face and related structures. ${ }^{2}$
The most frequent presenting symptoms of TMD are pain, TMJ sounds, tenderness in the masticatory muscles, headache, limited or asymmetric mandibular movement. Pain is usually localized in the muscles of mastication, preauricular area, or the TMJ. TMJ sounds are most frequently described as clicking, grating, or crepitus. ${ }^{3}$

TMDs requires meticulous examination and treatment planning. Various methods are used to diagnose TMDs, including radiographic measures, such as arthrography and tomography ${ }^{4}$, and methods that rely on the assessment of jaw movements.

\footnotetext{
* Lecturer, Orthodontic Department, Faculty of Oral and Dental Medicine, Ain Shams University, Cairo, Egypt.
} 
More recently, magnetic resonance imaging (MRI) has been used to evaluate the disk position. ${ }^{5}$ Cephalometric radiography can also be used to assess TMDs. ${ }^{6}$

The Research Diagnostic Criteria for Temporomandibular Disorders (RDC/TMD) is a diagnostic system for TMDs supported by a well-designed history and clinical examination protocol which provide specific reliable, reproducible and valid criteria with high sensitivity and specificity to define the most common types of TMDs. ${ }^{7}$

The data used to develop RDC/TMD came from longitudinal epidemiological research supported by the national institute for dental research (NIDR) which was conducted at the university of Washington. The RDC /TMD was designed to incorporate carefully documented and standardised sets of specifications for conducting a systematic and reliable clinical examination for TMD, operational definitions with demonstrated reliability of measurement for examination variablesRDC classifies TMD within two categories and allows multiple diagnoses to be made for a given patient: 1. Myofacial pain (muscle disorders) and 2. Disk displacements (joint disorders) ${ }^{8}$

Little is known about the risk factors for developing TMD, and the aetiology is a controversial issue. It is generally accepted that the etiology is multifactorial, and there are a large number of direct and indirect causal factors. TMD patients are often not only multisympomatic but also likely to exhibit more than one clinical sign of TMD. ${ }^{9}$

Although the etiology of the temporomandibular disorder is poorly understood and is said to be multifactorial, it is often debated that malocclusion is one of the risk factors for the disorder. Other risk factors being parafunctional habits and hyperlaxity of the joint ${ }^{10}$. Several theories are based on the presumption that there is an association and have justified the use of occlusal appliance therapy, anterior repositioning appliances, occlusal adjustments, orthodontic and orthognathic treatment. ${ }^{11-15}$

Occlusal interferences are a common finding in all age groups. Occlusal problems are not confined to static occlusion. This extends to involve functional occlusion with it's dynamic meaning and broader concept. Despite malocclusion is regarded as one of the major aetiological factors causing TMD, yet there are only a few weak and inconsistent correlations between occlusal interferences and signs and symptoms of TMD as well as for development of TMD. ${ }^{16}$

The aim of the study was to investigate the probable association between different types of TMDs and different types of malocclusion.

\section{MATERIALS AND METHODS}

One hundred participants complaining of TMJ problem were recruited from the clinic of surgery department ,Faculty of Dentistry, Ain shams university .

\section{Inclusion criteria:}

1- Adult participants, age range 19-45.

2- At least one symptom of TMJ problem is presented.

\section{Exclusion criteria:}

1- Any patient suffering from syndromes or systemic disease was excluded from this study.

2- Myofacial pain due to causes other than TMD.

\section{TMJ examination:}

For every participant, a thorough clinical examination was performed according to the Research Diagnostic Criteria for Temporomandibular Disorders (RDC/TMD) to confirm the TMD and classify it (Figure1). History taking included questions about 
whether the patient suffered from pain and its exact location. The opening pattern was then assessed by asking the patient to open and close slowly. This was used to assess presence or absence of lateral deviations. The participant was then asked to open maximally to assess the amount of opening he/she can perform painlessly, with pain and the maximum assisted opening.

Pain during protrusive and lateral excursions was assessed regarding it's presence or absence, exact location and degree. Joint sound was assessed during vertical and horizontal movements to identify its presence, type and location.

Muscle and joint palpation was then performed to identify the type of TMD; whether it is of muscle origin $(\mathrm{M})$, joint origin $(\mathrm{J})$ or combination of both(MJ). All facial extraoral and intraoral facial muscles were examined together with their origin, body and insertion. The joints were palpated from outside and inside the ears.

Pain was assessed using a visual analogue scale for pain (VAS) by giving the pain 10 degrees where zero is no pain and 10 is the maximum pain. The patient was asked to score the degree of pain on each palpated site and during different jaw movements.

The examination of all participants in the study was performed by a single calibrated examiner according to the guidelines RDC/TMD. The operator performed three palpations on each palpation site, and the most repeated score was reported. For all muscles that required the examiner to palpate at more than one site (eg: origin, body, insertion of masseter muscle), only the highest palpation score was taken.

\section{Occlusion examination:}

Full clinical orthodontic diagnosis was performed in both static and functional positions. Static occlusion examination included molar and canine classification, overjet, overbite, crossbites and any other malalignment (eg: rotation).

Full clinical examination of the functional movement was done where protrusive movement was evaluated regarding incised guidance and posterior clearance. Lateral excursions were examined for both balancing and working sides to determine the the presence or absence of lateral movement guidance (canine or group guidance) and the presence of interferences.

\section{Error Measurement}

Cohen's Kappa showed good inter-rater agreement in the assessment of occlusion problem $(0.82)$ and type of TMD problem(0.76).

\section{Statistical analysis}

Numerical data were explored for normality by checking the data distribution, calculating the mean and median values, evaluating histograms and normality curves. Numerical data was presented as mean and standard deviation categorical Data was presented as frequency and percentage. Fisher exact test and logestic regression were used to assess association between type of joint disorder and type of malocclusion. $\mathrm{T}$ test was used to compare between groups regarding age. Cohen,s Kappa was used to reveal agreement between raters assessment of occlusion problem and type of TMD problem.

\section{The significance level was set at $P \leq 0.05$.}

Statistical analysis was performed with IBM ${ }^{\circledR}$ SPSS $®$ Statistics Version 20 for Windows. 


\begin{tabular}{|c|c|c|c|c|c|c|c|c|c|c|}
\hline \multicolumn{11}{|c|}{ RDC- TMD Clinical Examination } \\
\hline Name & \multicolumn{3}{|c|}{ Date } & & & \multicolumn{5}{|c|}{ Examiner } \\
\hline \multicolumn{11}{|l|}{ 1. Review History } \\
\hline Presence of facial pain & & \multicolumn{2}{|c|}{0 NONE } & \multicolumn{2}{|c|}{ RIGHT } & & \multicolumn{2}{|c|}{2 LEFT } & \multicolumn{2}{|c|}{3 ВОТн } \\
\hline Location of facial pain & \multirow{2}{*}{$\begin{array}{l}\vec{E} \\
\overrightarrow{01}\end{array}$} & None & Muscle & Joint & Both & \multirow{2}{*}{ 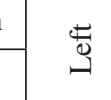 } & None & Muscle & Joint & Both \\
\hline & & 0 & 1 & 2 & 3 & & 0 & 1 & 2 & 3 \\
\hline
\end{tabular}

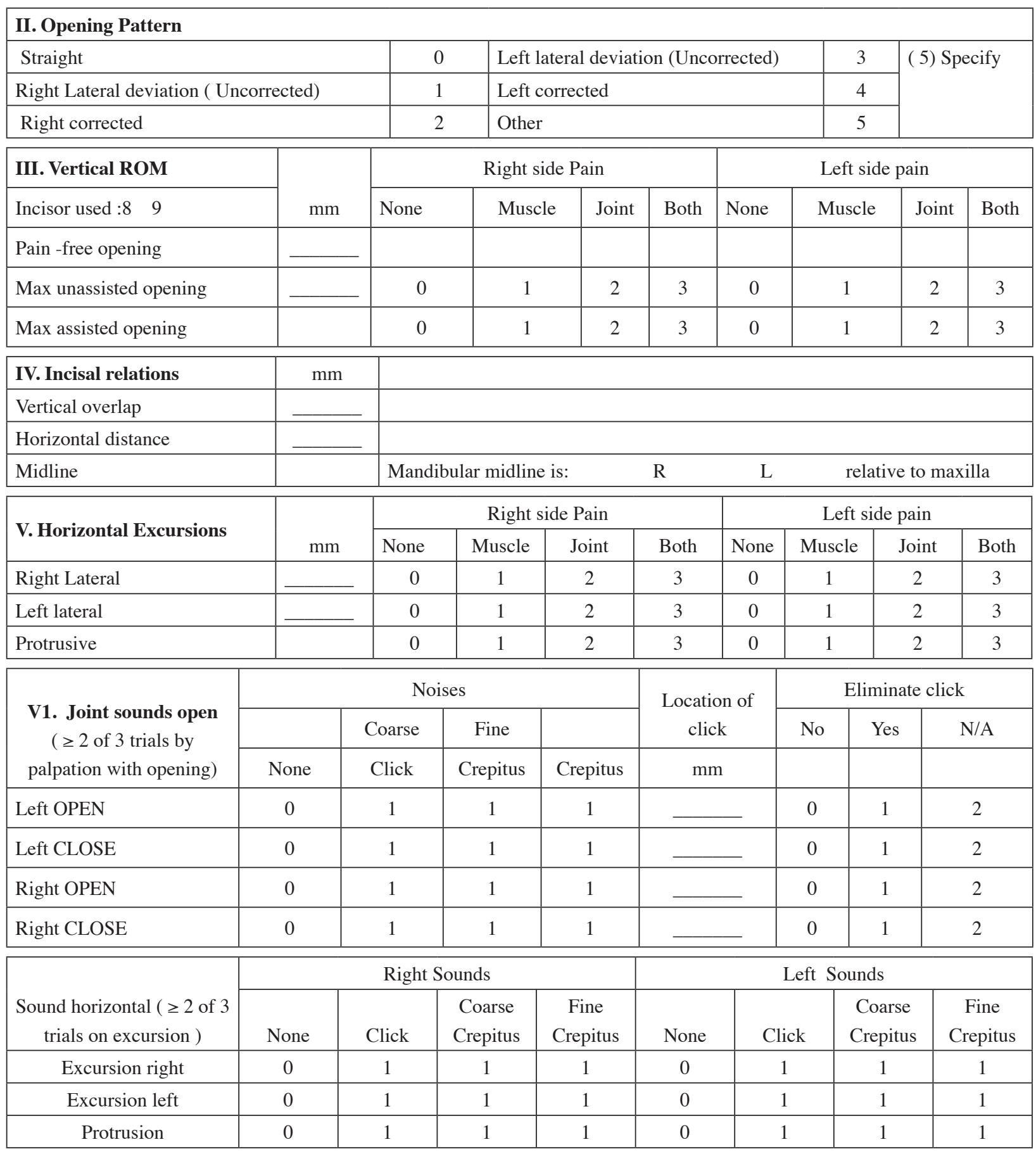




\begin{tabular}{|c|c|c|c|c|c|c|c|c|}
\hline \multicolumn{9}{|l|}{ VII. Muscle and joint pain with palpation } \\
\hline & \multicolumn{4}{|c|}{$\begin{array}{c}\text { RIGHT } \\
\text { RDC protocol }\end{array}$} & \multicolumn{4}{|c|}{$\begin{array}{c}\text { LEFT } \\
\text { RDC protocol }\end{array}$} \\
\hline & no & mid & $\bmod$ & sevr & no & mid & $\bmod$ & sevr \\
\hline \multicolumn{9}{|l|}{ Non pain sites } \\
\hline Mastiod (lateral superior potion) & 0 & 1 & 2 & 3 & 0 & 1 & 2 & 3 \\
\hline Frontal (in line with pupil, below hair) & 0 & 1 & 2 & 3 & 0 & 1 & 2 & 3 \\
\hline Vertex ( $1 \mathrm{~cm}$ lateral to cranial top) & 0 & 1 & 2 & 3 & 0 & 1 & 2 & 3 \\
\hline \multicolumn{9}{|l|}{ Extra \& cervical muscles } \\
\hline Posterior temporalis (back of temple) & 0 & 1 & 2 & 3 & 0 & 1 & 2 & 3 \\
\hline Middle temporalis ( Middle of temple) & 0 & 1 & 2 & 3 & 0 & 1 & 2 & 3 \\
\hline Anterior temporalis ( frontal of temple) & 0 & 1 & 2 & 3 & 0 & 1 & 2 & 3 \\
\hline Masseter origin ( cheek under cheekbone) & 0 & 1 & 2 & 3 & 0 & 1 & 2 & 3 \\
\hline Masseter body ( cheek side of face) & 0 & 1 & 2 & 3 & 0 & 1 & 2 & 3 \\
\hline Masseter insertion ( cheek jaw line) & 0 & 1 & 2 & 3 & 0 & 1 & 2 & 3 \\
\hline Post mandibular region ( Jaw throat area) & 0 & 1 & 2 & 3 & 0 & 1 & 2 & 3 \\
\hline Submandibular region ( under chin) & 0 & 1 & 2 & 3 & 0 & 1 & 2 & 3 \\
\hline \multicolumn{9}{|l|}{ Joint pain } \\
\hline Lateral pole (outside) & 0 & 1 & 2 & 3 & 0 & 1 & 2 & 3 \\
\hline Posterior attachment (inside ear) & 0 & 1 & 2 & 3 & 0 & 1 & 2 & 3 \\
\hline \multicolumn{9}{|l|}{ Intra oral muscle } \\
\hline Lat pterygoid area (behind upper molar) & 0 & 1 & 2 & 3 & 0 & 1 & 2 & 3 \\
\hline Tendon of temporalis (tendon) & 0 & 1 & 2 & 3 & 0 & 1 & 2 & 3 \\
\hline
\end{tabular}

Figure (1): TMD clinical examination form

\section{RESULTS}

Gender was equally distributed between the muscle (M) group and the combined muscle and joint (MJ) group. Fisher exact test showed no statistical significant difference between groups. Mean value of age for M group was 27.3 and for MJ group was 25.7. However $t$ test showed no statistical difference between groups. M group showed higher prevalence of normal occlusion, static malocclusion, and functional malocclusion, however regarding combined static and functional problems, MJ group showed higher prevalence. Fisher exact test showed correlation between type of TMD (muscle or muscle and joint) and type of malocclusion Table(I).

Logestic regression showed association between static and functional malocclusion group and type of joint disorder as the combined static and functional malocclusion group showed 16.67 odds in comparison to no occlusal problem group to have combined muscle and joint TMD problem Table (II). 
TABLE (I) Gender, age and type of occlusion distribution among TMD groups and correlation between type of TMD ( $\mathrm{M}$ or MJ) and type of malocclusion (Static and Functional).

\begin{tabular}{|c|c|c|c|}
\hline & \multicolumn{2}{|c|}{ TMD } & \multirow{2}{*}{$\begin{array}{c}P \\
\text { value }\end{array}$} \\
\hline & M & MJ & \\
\hline \multicolumn{4}{|l|}{ Gender } \\
\hline Male $\mathrm{n}(\%)$ & $3(50)$ & $3(50)$ & \multirow[t]{2}{*}{1} \\
\hline Female $\mathrm{n}(\%)$ & $47(50)$ & $47(50)$ & \\
\hline Age & & & \multirow{3}{*}{0.178} \\
\hline \multirow[t]{2}{*}{ Mean (std.) } & 27.3 & 25.73 & \\
\hline & $(5.02)$ & $(6.48)$ & \\
\hline \multicolumn{4}{|l|}{ Occlusion } \\
\hline Normal $\mathrm{n}(\%)$ & $16(72.7)$ & $6(27.3)$ & \multirow[t]{4}{*}{$<0.001$} \\
\hline Static n $(\%)$ & $4(80)$ & $1(20)$ & \\
\hline Functional $\mathrm{n}(\%)$ & $26(60.5)$ & $17(39.5)$ & \\
\hline Static and functional $\mathrm{n}(\%)$ & $4(13.3)$ & $26(86.7)$ & \\
\hline
\end{tabular}

TABLE (II) Association between type of TMD and type of malocclusion.

\begin{tabular}{l|c|c|c|c|}
\cline { 3 - 4 } & \multirow{2}{*}{$\operatorname{Exp}(\mathrm{B})$} & \multicolumn{2}{|c|}{$95 \%$ C.I.for $\operatorname{EXP}(\mathrm{B})$} & \multirow{2}{*}{ P value } \\
\cline { 3 - 4 } & & Lower & Upper & \\
\hline No (reference) & & & $<0.001$ \\
Static & .667 & .061 & 7.230 & 0.739 \\
Functional & 1.889 & .613 & 5.820 & 0.268 \\
Static and func- & 16.667 & 4.060 & 68.417 & $<0.001$ \\
tional & .375 & & & 0.04 \\
Constant & & & \\
\hline
\end{tabular}

\section{DISCUSSION}

Radiographic imaging has long been used as the examination of choice for TMJ evaluations in orthodontic practice. Cone beam computed tomography (CBCT) was preferred over CT images since it provides high-resolution imaging, diagnostic reliability, and $40 \%$ less radiation than conventional CT. ${ }^{17}$
The invasiveness of radiography and the costintensiveness of other imaging techniques (eg, magnetic resonance imaging [MRI]), favored clinical examinations as a diagnostic tool of TMDs. Several clinical procedures have been used to evaluate the status of the TMJ. However, there was a necessitating demand on the validity and reliability of a diagnostic system. This is mandatory for correct classification of the status of the TMJ.

RDC offers an alleged diagnostic and classification system in which the reliability, validity and clinical usefulness for TMD diagnosis and classification could be scientifically evaluated. It can also be revised using an evidence-based model for successive repetitions. ${ }^{?}$

An important feature of RDC is that it distinguishes the type of TMD, whether due to muscle origin or joint origin. This was long overlooked in many previous studies that used the radiographic methods for TMD diagnosis $4,6,17$ The radiographic method only showed joint problems without efficiently delineating muscular problems.

Differentiating static and functional occlusion was a mandate to overcome the pitfalls of previous studies correlating TMD and malocclusion. Narrowing the diagnosis of malocclusion to static occlusion- where open bite and crossbites gained most of the attention- compromised the results regarding the TMD patient suffering malocclusion. In some instances patients have functional malocclusions despite the normal static occlusion and thus should be classified as malocclusion cases.

Not only was functional occlusion assessed, but it was also assessed from all its aspects; the balancing side as well as the working side. The balancing side interference is an important perspective of malocclusion that is anticipated to be a probable factor in TMD problems.

The studied sample composed of adult participants. This is to avoid pain occurring in the children 
that might be independent of other complaints or objective signs of problems in the TMJ region.

Alterations in occlusion such as malalignment, crossbite, open-bite, occlusal interferences, increased overjet and deep bite, crowding and missing teeth have been identified in different studies as triggering factors. ${ }^{18,19}$ There seems to be a weak association between occlusal factors and TMD .

In the view of the information provided by the literature and studies, the precise role of occlusion in TMJ pathology does not seem to be clearly defined. The proof linking the malocclusion to the temporomandibular disorder should have several criteria as suggested by Hill in $1965 .{ }^{20}$ There comes in the first place that the effects should follow the cause, whereby in literature and studies, it is shown to be the opposite where for example muscle pain causes a change in occlusion. Additionally, speaking on the evidence level, the scientific literature should be consistent. But this does not apply to temporomandibular disorders. The publications from 1995 through 2016 state that there increasing numbers of studies that downgrade the importance of the role of occlusal factors in the etiology of TMD.

In our study, malocclusion was more prevalent in patients having combined muscle and joint problem than those having only muscle problem. There was a statistically significant correlation between TMD and combined static and functional malocclusions. While there was no correlation between TMD and static malocclusion or TMD and functional malocclusion. Participants with static and functional malocclusions were at 16.6 higher risk to have muscle and joint problem than participants with normal occlusion. This throws a light on the strength of association between TMD and malocclusion.

These results are in accordance with a study published in the year 2005, where comparison was made of a group of women with internal derangement of the temporomandibular joint versus women who were asymptomatic control. ${ }^{6}$ The subjects with disc displacement were characterized by unilateral posterior crossbite and long displacement of centric relation to the position of maximum intercuspation. The authors concluded that occlusal alterations may be a cofactor in the identification of patients with TMD. They stated that some differences in occlusion may be the result rather than an etiology of TMD.

However,the results are contradictory to a study carried out by Hirsh et.al, who after studying a total of 3033 subjects concluded that greater or lesser overjet or overbite even at extreme values does not constitute a risk factor for the presence of joint sounds, such as reciprocal clicks and crepitation. ${ }^{21}$

In other work published by Magnusson et al which involved the follow-up of 402 patients over a period of 20 years, has concluded that occlusal factors are weakly associated with temporomandibular disorder. $^{22}$

The emphasis on diagnosing TMDs of muscle origin together with joint origin and the detailed examination of functional occlusion side by side with static occlusion in this study, might be a tribute to the correlation found between TMDs and malocclusion.

It is important to point out that these results showed only association between TMD types and malocclusion types. A causal relationship needs to be investigated by clinical trials where a temporal relation is a mandate to reveal the presence or absence of this relation.

\section{CONCLUSION}

The type of TMD whether muscle or joint problem is an important factor when correlating TMD to malocclusion. Combined muscle and joint problems are correlated to malocclusion of combined static and functional occlusal problems. 


\section{REFERENCES}

1- Gidarakou IK, Tallents RH, Kyrkanides S, Stein S, Moss ME. Comparison of Skeletal and Dental Morphology in Asymptomatic Volunteers and Symptomatic Patients with Normal Temporomandibular Joints. Angle Orthod 2003;73:116-120.

2- Michelotti A, Iodice G. The Role of orthodontics in temporomandibular disorders, Review Article. Journal of Oral Rehabilitation 2010 37; 411-429.

3- Okeson JP. Orofacial pain: guidelines for assessment, diagnosis and management. Quintessence Publishing Co. 1996:116

4- Katzberg RW, Westesson PL, Tallents RH, Drake CM. Orthodontics and temporomandibular joint internal derangement. Am J Orthod Dentofacial Orthop. 1996; 109:515-520.

5- Tallents RH, Katzberg RW, Murphy W, Proskin H. Magnetic resonance imaging findings in asymptomatic volunteers and symptomatic patients with temporomandibular disorders. J Prosthet Dent. 1996;75:529-533.

6- Ahn SJ, Kim TW, and Nahm DS . Cephalometric keys to internal derangement oftemporomandibular joint in women with Class II malocclusions. Am J Orthod Dentofacial Orthop 2004;126:486-95

7- Dworkin SF. Research Diagnostic Criteria for Temporomandibular Disorders: current status and future relevance. Journal of Oral Rehabilitation 2010 37; 734-743.

8- Dworkin SF, LeResche L (eds.). Research Diagnostic Criteria for Temporomandibular Disorders: review, criteria, examinations and specifications, critique. J Craniomandib Disord. 1992;6:301-355.

9- Magnusson T, Egermark I, Carlsson GE. A prospective investigation over two decades on signs and symptoms of temporomandibular disorders and associated variables. A final summary. Acta Odontologica Scandinavia, 2005; 63:99

10- Poveda-Roda R, Bagan JV, Diaz-Fernandez JM, Hernandez-Bazan S, Jimenez-Soriano Y, Review of temporomandibular joint pathology. Part 1: Classification, Epidemiology and risk factors. Med Oral Patol Oral Cir Bucal 2007;12:E292-8.
11- Ekberg E, Vallon D, Nilner M. The efficacy of appliance therapy in patients with temporomandibular disorders of mainly myogenous origin. A randomized, controlled, short-term trial. J Orofac Pain. 2003;17:133-139.

12- Kurita H, Ohtsuta A, Kurashina K, Kopp S. A study of factors for successful splint capture of anteriorly disc displaced Temporomandibular joint disc with disc repositioning appliance. J Oral Rehab. 2001;28:651-657.

13- De Boever JA, Carlsson GE, Klineberg IJ. Need for occlusal therapy and prosthodontic treatment in the management of temporomandibular disorders. Part I. Occlusal interferences and occlusal adjustment. J Oral Rehab. 2000;27:367-379.

14- Sadowsky C, Theisen TA, Sakols EI. Orthodontic treatment and temporomandibular joint sounds -a longitudinal study. Am J Orthod Dentofacial Orthop. 1991;99:441-447.

15- Egermark I, Blomqvist JE, Cromvik U, Isaksson S. Temporomandibular dysfunction in patients treated with orthodontics in combination with orthognathic surgery. Eur J Orthod. 2000;22:537-544.

16- Ramfjord SP. Bruxism, a clinical and electromyographic study. J Am Dent Assoc. 1961;62:21-44.

17- Vitral RWF, Campos MJD, Rodrigues AF, Fragac MR. Temporomandibular joint and normal occlusion: Is there anything singular about it? A computed tomographic evaluation. Am J Orthod Dentofacial Orthop 2011;140:18-24.

18- M.Zhang, C.Mcgrath, U.Hagg. The impact of malocclusion and its treatment on quality of life: A literature review. International Journal of Paediatric Dentistry 2006; 16: $381-387$

19- Obrez A, Stohler CS. Jaw muscle pain and its effects on gothic arch tracing. J Prosthet Dent. 1996;75:393-398.

20- Hill AB. The environment and disease: association or causation? Proc R Soc Med 1965;58:295-300.

21- Hirsch C, John MT, Drangsholt MT, Mancl LA. Relationship between overbite / overjet and clicking or crepitus of the temporomandibular joint. J Orofac Pain. 2005;19:218-225.

22- Magnusson T, Carlsson GE, Egermark I. Changes in clinical signs of craniomandibular disorders from the age of 15 to 25 years. J Orofac Pain. 1994;8:207-215. 